Canadian Oncology

Nursing Journal

Revue canadienne

de soins infirmiers

en oncologie

Volume 31, Issue 3 • Summer 2021

elSSN: 2368-8076 


\title{
Connaissances, attitudes et croyances concernant le dépistage du cancer du col utérin dans le District d'Ajumako-Enyan-Essiam au Ghana
}

\author{
par Charlotte Naa Sampson, Samuel Donkor Nkpeebo, Thywill Amenuveve Degley
}

\section{RÉSUMÉ}

L'étude a été menée dans le district d'Ajumako-Eyan-Essiam (AEED) et visait à évaluer les connaissances, attitudes et croyances des femmes relativement au dépistage du cancer du col utérin. Au Ghana, ce cancer vient au deuxième rang des causes de cancer gynécologique. Dans les études et la pratique clinique effectuées auprès de femmes atteintes de cancer du col utérin, le diagnostic précoce, le traitement et la prévention du cancer sont largement influencés par leurs connaissances et leurs attitudes envers le dépistage. À ce jour, on ne dispose pas d'étude sur ces perceptions chez les femmes dans le district d'AEED. Un devis transversal quantitatif a été utilisé avec une analyse statistique descriptive de données provenant de 240 femmes. Les résultats ont révélé que 61,3\% d'entre elles ne savaient pas quel organe était touché lors d'un cancer du col utérin ou à quel intervalle un suivi était nécessaire après un résultat de test Pap normal; il $y$ aurait donc des lacunes dans les connaissances. Au total, $36 \%$ croyaient que le test de Papanicolaou (test Pap) était effectué une fois pour toutes, et 57,1\% pensaient qu'il était dispendieux, ce qui limite la participation au dépistage. Par peur de l'inconnu, beaucoup de répondantes avaient refusé le dépistage $(48,8 \%)$ alors que d'autres croyaient qu'elles n'étaient pas à risque (65,8\%). De plus, bon nombre de femmes (46,7\%) pensaient qu'il n'y avait pas de traitement contre le cancer du col. En conclusion, les connaissances fausses et inadéquates de ces femmes ont influencé leurs attitudes envers le dépistage du cancer.

\footnotetext{
AUTEURS

Charlotte Naa Sampson, Enseignante, Nursing and Midwifery Training College, Teshie, TS861, Accra, Ghana, cdasampson18@gmail.com

Samuel Donkor Nkpeebo, Chargé de cours adjoint, Family Health University College, Teshie, Accra, Ghana, samdonkor.nkpeebo@gmail.com

Thywill Amenuveve Degley, Coordonnatrice clinique, Stanford World Clinic, Cape Coast, Ghana, tamenuveve@gmail.com

DOI:10.5737/23688076313291297
}

Mot-clés : cancer du col utérin; connaissances, dépistage, attitude, croyances en matière de santé; traitement, test Pap, virus du papillome humain (VPH)

\section{INTRODUCTION}

T e cancer du col utérin est le troisième cancer gynécoloـ gique le plus répandu à travers le monde, avec une estimation de 569847 nouveaux cas et 311365 décès en 2018 (Bruni et al., 2019). Environ $83 \%$ des cas surviennent dans les pays en développement, où les femmes ne vont consulter que lorsqu'elles sont à un stade avancé et que seuls des traitements palliatifs sont envisageables. Au Nigéria, on diagnostique approximativement 14943 nouveaux cas de cancer du col utérin chaque année (estimation pour 2018). Au Ghana, 3151 nouveaux cas de ce cancer sont diagnostiqués annuellement (estimation pour 2018), ce qui en fait le deuxième cancer en importance chez les femmes après le cancer du sein (Bruni et al., 2019). Dans ces deux pays, l'écart entre les nombres de cas réside dans la taille de la population; au Nigéria, la population atteint 209 millions dont 21,3\% sont des femmes de 18 à 64 ans (Adenola et al., 2017). Le Ghana a une population totale de 24658823 (26,5\%), dont 6530900 sont des femmes de 18 à 64 ans (Ghana demographics, 2015). Malgré ces statistiques marquantes, la prévention du cancer du col utérin n'est pas souvent évoquée au Ghana.

On estime que $95 \%$ des femmes n’ont jamais subi de dépistage du cancer du col utérin dans les pays en développement (Tung et al., 2010), et que seulement 2,1 \% des Ghanéennes ont déjà subi un test Pap (Adanu, Seffah, Duda, Darko, Hill et Anarfi, 2020). La plupart du temps, des programmes de dépistage efficaces permettront de détecter ce cancer, car la fréquence du cancer du col utérin et les décès associés diminuent considérablement lorsqu'un programme systématique de dépistate par test Pap existe. Au Ghana, comme les services de dépistage ne sont offerts qu'à certains endroits, surtout dans les centres urbains, les patientes doivent, la plupart du temps, se déplacer pour en profiter. Dans une étude réalisée dans l'Est ougandais par Mukama (2017), la plupart des femmes avaient peu de connaissances sur le dépistage et sur ce service. De plus, même après avoir subi le test, elles ne se présentaient pas au rendez-vous de suivi et aux soins ultérieurs si une anomalie était détectée lors du dépistage. Pour que le dépistage soit vraiment utile, les femmes doivent savoir que ce service est offert et connaître la maladie, et il faut aussi démystifier les fausses croyances sur la santé. Tout ceci a également été confirmé par Ebu et collaborateurs (2015), qui ont indiqué que les croyances des femmes sur la santé ainsi que leurs connaissances et leurs 
attitudes seraient la cause de la faible participation au dépistage au Ghana. Par conséquent, ce cancer demeure un enjeu de santé publique.

De plus, on a rapporté que comprendre les comportements liés à la santé selon le contexte culturel est d'une importance capitale si l'état de santé d'un groupe à risque doit être amélioré (Mattocks, Kauth, Sandfort, Matza, Sullivan et Shipherd, 2014). Par ricochet, explorer la base des connaissances des femmes ainsi que leurs attitudes et croyances en matière de santé liée au dépistage du cancer du col utérin aidera probablement à augmenter l'adhésion au dépistage et à réduire la morbidité et la mortalité associées à cette maladie. Cela aiderait aussi à susciter l'intérêt d'autres chercheurs à mener des recherches dans ce domaine afin d'ajouter leur conclusion au corpus de connaissances.

Létude visait à analyser l'effet des connaissances, de l'attitude et des croyances en matière de santé des femmes sur la participation au dépistage du cancer du col utérin dans le district d'Ajumako-Enyan-Essiam dans le sud du Ghana.

\section{MÉTHODOLOGIE}

Létude a été réalisée dans le district d'Ajumako-EnyanEssiam, situé dans la République du Ghana. Ajumako-EnyanEssiam (Ghana Districts, 2006) est l'un des dix-sept districts de la région du Centre du Ghana. Il s'agit d'une zone rurale couvrant 540,3 kilomètres carrés et représentant $5 \%$ de la région du Centre. Le district d'Assin borde la frontière à l'ouest, tandis que le district de Mfantsiman est situé au sud, le district d'Asikuma-Odoben-Brakwa (AOB), au nord-ouest, et le district de Gomoa, à l'est (Ghana Districts, 2006). Sa projection démographique est de 138046 personnes et comprend 73628 femmes (Demographic and Health Survey, 2015). On trouve dans ce district 169 communautés.

Létude fait appel à un devis transversal, qui permet au chercheur de recueillir des données sur une population ou un groupe défini de façon ponctuelle ou durant une période donnée. La collecte de données a duré quatre mois, soit de juillet à novembre 2019.

Le groupe à l'étude comptait des femmes de 18 à 64 ans vivant dans la région du district d'Ajumako-Enyan-Essiam. En raison de l'inconfort associé à la grossesse, les femmes enceintes n'étaient pas admises à l'étude puisqu'elles vivent déjà d'autres symptômes et que le test aurait été ardu à réaliser et inconfortable pour elles. De plus, le risque d'infection est très élevé pour ces femmes. Les femmes qui avaient déjà eu un cancer du col utérin n'étaient pas admises non plus.

L'étude a utilisé une procédure d'échantillonnage non probabiliste de stratification et de commodité pour déterminer la taille de l'échantillon à l'aide des procédures d'échantillonnage qui suivent. Premièrement, les i69 municipalités du district ont été stratifiées en quatre groupes principaux sur la base des frontières de districts : régions nord, sud, est et ouest (Ghana Districts, 2006). Après la stratification du district, on a sélectionné deux localités dans chaque strate avec une technique d'échantillonnage aléatoire simple de méthode par vote. Au total, huit localités ont été sélectionnées. Un échantillonnage de commodité a ensuite été fait dans chaque localité pour sélectionner les participantes. La taille de l'échantillon total $(\mathrm{N}=204)$ sélectionné dans le cadre de l'étude se fonde sur la formule statistique de Glenn (1992). La taille de l'échantillon a été augmentée à 240 afin de compenser la non-réponse. Parmi les 240 répondantes, il y en avait environ 30 de chaque localité.

Un questionnaire auto-administré a été élaboré sur les connaissances, l'attitude et les croyances en matière de santé. L'outil a été conçu aux fins de l'étude et a fait l'objet de discussions avec un superviseur ayant les connaissances nécessaires dans le domaine. Les questions visaient à recueillir de l'information auprès des répondantes sur les connaissances, l'attitude et les croyances en matière de santé sur le dépistage du carcinome du col utérin.

Pour veiller à l'efficacité et à l'efficience des procédures de collecte de données, des infirmières qui parlaient le dialecte local ont été embauchées dans chaque localité pour travailler avec le chercheur afin d'aider les répondantes à entreprendre efficacement cette collecte. Avant l'embauche de ces infirmières comme personnel de recherche, des rencontres ont eu lieu pour veiller à ce qu'elles comprennent les objectifs de l'étude et le rôle attendu.

On a approché les femmes des localités, et on leur a expliqué l'étude et le questionnaire. Celles qui ont accepté de participer ont eu le temps de répondre dans la même journée. Les questionnaires ont été placés dans une enveloppe pré-adressée avec une lettre de présentation ainsi qu'un stylo en guise de remerciement afin d'inciter la participation à l'étude. Chaque répondante a signé un formulaire de consentement, après avoir reçu des explications détaillées pour garantir son droit à l'autodétermination et à l'autonomie. Les répondantes ont rempli le questionnaire puis ont scellé l'enveloppe afin de préserver la confidentialité des données. Le chercheur principal a expliqué le projet et distribué le formulaire, puis le personnel de l'étude a circulé parmi les répondantes pour veiller à ce qu'elles remplissent adéquatement le questionnaire. Les questionnaires remplis ont ensuite été collectés par le chercheur et le personnel.

Les données colligées ont été analysées avec Statistical Package for Social Sciences (SPSS), et les statistiques descriptives ont été utilisées pour déterminer la fréquence des réponses à chaque question.

\section{RÉSULTATS}

\section{Niveau de connaissances sur le test Pap}

Léchantillon était composé de 240 femmes de 18 à 64 ans venant du district d'Ajumako-Enyan-Essiam. Un peu plus de deux répondantes sur cinq (41,7\%) avaient entre 21 et 30 ans; 39,6\% avaient entre 31 à 40 ans; et 8,2\% avaient de 51 à 60 ans. Pour ce qui est de l'état civil, 51,7 \% étaient des femmes mariées, mais 2,9\% étaient divorcées ou séparées. Un peu plus de la moitié $(53,3 \%)$ des femmes avaient fait des études, 24,2\%, des études postsecondaires.

Pour ce qui est des connaissances, la majorité des répondantes (74 \%) ont rapporté qu'elles avaient entendu parler $\mathrm{du}$ test Pap. Cependant, seulement 47,5 \% des femmes sondées savaient qu'un résultat normal signifiait que le risque 


\begin{tabular}{|c|c|c|}
\hline \multicolumn{3}{|c|}{$\begin{array}{l}\text { Tableau } 1 \text { : Connaissances des femmes sur le cancer du col utérin et } \\
\text { le test de Papanicolaou (test Pap) }\end{array}$} \\
\hline Connaissances sur le cancer utérin & Fréquence & Pourcentage \\
\hline \multicolumn{3}{|l|}{ Le cancer du col utérin est: } \\
\hline Le cancer de l'utérus & 58 & 24,2 \\
\hline Le cancer des ovaires & 14 & 5,8 \\
\hline Le cancer du vagin & 147 & 61,3 \\
\hline Le cancer des trompes & 9 & 3,8 \\
\hline Je ne sais pas & 12 & 5,0 \\
\hline \multicolumn{3}{|l|}{ Principale cause du cancer du col utérin : } \\
\hline Relations sexuelles précoces & 26 & 10,8 \\
\hline Partenaires sexuels multiples & 143 & 59,6 \\
\hline Multiparité & 33 & 13,8 \\
\hline Statut socio-économique faible & 3 & 1,3 \\
\hline $\begin{array}{l}\text { Infection par le virus du papillome } \\
\text { humain }\end{array}$ & 35 & 14,6 \\
\hline \multicolumn{3}{|l|}{ Avez-vous déjà entendu parler du test Pap? } \\
\hline Oui & 178 & 74,0 \\
\hline Non & 62 & 36,0 \\
\hline \multicolumn{3}{|l|}{ Un test Pap normal signifie: } \\
\hline Risque faible de développer la maladie & 114 & 47,5 \\
\hline Aucun risque de développer la maladie & 60 & 25,0 \\
\hline Je n'ai pas besoin d'autres tests Pap & 28 & 11,7 \\
\hline Je ne sais pas & 38 & 15,8 \\
\hline \multicolumn{3}{|l|}{$\begin{array}{l}\text { Quel est l'intervalle du suivi après un } \\
\text { résultat normal? }\end{array}$} \\
\hline 6 mois & 140 & 58,3 \\
\hline 2 mois & 55 & 22,9 \\
\hline Un an & 8 & 3,3 \\
\hline Trois ans & 0 & 0,0 \\
\hline Je ne sais pas & 37 & 15,4 \\
\hline \multicolumn{3}{|l|}{$\begin{array}{l}\text { Risques pour celles qui prennent des } \\
\text { contraceptifs }\end{array}$} \\
\hline Oui & 122 & 50,8 \\
\hline Non & 92 & 38,3 \\
\hline Je ne sais pas & 26 & 10,8 \\
\hline \multicolumn{3}{|l|}{ Qui effectue le test Pap? } \\
\hline Infirmière & 28 & 11,7 \\
\hline Omnipraticien & 30 & 12,5 \\
\hline Gynécologue & 182 & 75,9 \\
\hline \multicolumn{3}{|l|}{$\begin{array}{l}\text { À quel âge peut-on subir un test de } \\
\text { dépistage du cancer du col utérin? }\end{array}$} \\
\hline $18-25$ & 78 & 32,5 \\
\hline $26-35$ & 59 & 24,6 \\
\hline $36-60$ & 49 & 20,4 \\
\hline 70 ans et moins & 12 & 5,0 \\
\hline Je ne sais pas & 42 & 17,5 \\
\hline Total & 240 & 100 \\
\hline
\end{tabular}

de développer la maladie était faible; $25 \%$ croyaient qu'il n'y avait pas de risque de la développer. Il convient de noter que $15,8 \%$ ne savaient pas ce que signifiait un résultat normal suite au test Pap. Au total, 58,3\% des répondantes ont indiqué qu'il devait y avoir un suivi dans les six mois suivant un résultat normal, mais $22,9 \%$ ont indiqué que cétait dans les deux mois. Cependant, 15,4 \% ne savaient pas quel était le délai approprié pour un suivi (voir tableau 1).

Parmi les répondantes, $35 \%$ ont mentionné le VPH comme la principale cause du cancer du col utérin, mais plus de la moitié $(59,6 \%)$ l'ont attribué à de multiples partenaires sexuels. La moitié des répondantes $(50,8 \%)$ ont rapporté qu'il y avait des risques accrus chez les femmes qui prenaient des contraceptifs hormonaux, mais 10,8\% ne savaient pas si elles étaient à risque ou non. La majorité $(75,9 \%)$ était d'avis que le test Pap devait être réalisé par un gynécologue. Dans cette étude, environ le tiers des répondantes $(32,5 \%)$ ont indiqué que les femmes étaient susceptibles de développer un cancer du col utérin entre 18 et 25 ans, mais $24,6 \%$ ont plutôt indiqué entre 26 et 35 ans. Cependant, 17,5\% d'entre elles ne savaient pas à quel âge les femmes pouvaient développer un cancer du col (voir tableau 1).

\section{Expérience du dépistage du cancer du col utérin}

Comme le montre le tableau $2 a$, le quart $(25,4 \%)$ des répondantes avaient déjà passé un test Pap, contre 73,3 \% qui n'en avaient jamais eu. Seules $6,3 \%$ des répondantes ont indiqué qu'elles avaient elles-mêmes décidé de passer le test, mais $5 \%$ s'y étaient rendues parce que leur médecin l'avait conseillé. La plupart des répondantes $(65 \%)$ ont indiqué qu'elles préféraient passer le test dans un hôpital, mais $20 \%$ ont dit qu'elles préféraient se rendre dans un centre de dépistage. Les autres ont mentionné n'avoir aucune préférence. La majorité des répondantes pensaient que le cancer du col utérin était dangereux. Cependant, $11,7 \%$ ne savaient pas si cétait bénin ou dangereux (voir tableau 2a).

\section{Attitude des répondantes par rapport au dépistage}

Parmi les participantes, 67,5\% ont mentionné que le dépistage du cancer du col utérin n'était pas utile. Par contre, presque toutes $(98,8 \%)$ se sont montrées intéressées au test de dépistage. Elles ont aussi toutes affirmé qu'elles encourageraient d'autres femmes à se soumettre au dépistage. Seulement 9,6\% des répondantes ont rapporté que leur religion était contre ce dépistage, et $96,7 \%$ ont indiqué que ce n'était pas permis en raison de leur culture. La majorité d'entre elles $(72,5 \%)$ ont affirmé que penser au cancer les rendait craintives, même si $27,9 \%$ des participantes pensaient être susceptibles de le développer. Parmi les répondantes, 82,9\% sentaient que le dépistage était important (voir le tableau $2 \mathrm{~b}$ ).

\section{Croyances en matière de santé sur le dépistage du cancer du} col utérin

Le tiers $(34,2 \%)$ des répondantes croyaient qu'elles pouvaient avoir des lésions précancéreuses, alors que 24,6\% n'en savaient rien. La majorité (95\%) considérait que le cancer du col utérin était un enjeu de santé publique. La moitié des participantes (49,6\%) étaient d'avis qu'il était possible de guérir 
ce cancer. La majorité des répondantes (96,7\%) ne considérait pas le cancer du col utérin comme une malédiction, et $90,4 \%$ ont dit qu'elles se sentiraient soulagées d'avoir passé le test Pap. Les répondantes ont indiqué à 78,8 \% qu'un résultat normal leur donnait un sentiment de contrôle, et 79,6 \% croyaient que le test devrait être effectué régulièrement (voir le tableau 3).

\section{DISCUSSION}

Dans la présente étude, la plupart des répondantes ont rapporté avoir déjà entendu parler du dépistage du cancer $d u$ col utérin. Ce résultat est d'une importance fondamentale, car avoir des connaissances sur la prévention liée à la santé influence positivement l'attitude et la pratique chez ces personnes et facilite l'acceptation et la promotion de lignes de conduite qui ont à voir avec ces maladies. Dans d'autres études, l'évaluation des connaissances ou la sensibilisation chez la population générale a révélé que les gens manquent souvent

\begin{tabular}{|c|c|c|}
\hline \multicolumn{3}{|c|}{$\begin{array}{l}\text { Tableau } 2 \mathrm{a} \text { : Expérience des répondantes sur le dépistage du } \\
\text { cancer du col utérin }\end{array}$} \\
\hline $\begin{array}{l}\text { Expérience du dépistage du cancer du } \\
\text { col utérin }\end{array}$ & $\begin{array}{l}\text { Fréquence } \\
\text { N (240) }\end{array}$ & $\begin{array}{l}\text { Pourcentage } \\
\text { (\%) }\end{array}$ \\
\hline \multicolumn{3}{|l|}{ Avez-vous déjà subi un test Pap? } \\
\hline Oui & 61 & 25,4 \\
\hline Non & 176 & 73,3 \\
\hline Je ne sais pas & 3 & 1,3 \\
\hline \multicolumn{3}{|l|}{$\begin{array}{l}\text { À quelle fréquence doit-on subir un } \\
\text { test Pap? }\end{array}$} \\
\hline Une fois par année & 27 & 11,3 \\
\hline Tous les trois ans & 12 & 5,0 \\
\hline Tous les cinq ans & 0 & 0,0 \\
\hline Tous les 10 ans & 6 & 2,5 \\
\hline Ne s'applique pas & 195 & 81,3 \\
\hline \multicolumn{3}{|l|}{ Raison du dépistage (test Pap) } \\
\hline Initiative personnelle & 15 & 6,3 \\
\hline Demandé par le médecin & 12 & 5,0 \\
\hline Dépistage systématique & 12 & 5,0 \\
\hline Autre raison & 6 & 2,5 \\
\hline Ne s'applique pas & 195 & 81,3 \\
\hline \multicolumn{3}{|l|}{$\begin{array}{l}\text { Impressions concernant le dépistage } \\
\text { du cancer du col }\end{array}$} \\
\hline Bonne & 23 & 9,6 \\
\hline Mauvaise & 189 & 78,8 \\
\hline Pas d'opinion & 28 & 11,7 \\
\hline
\end{tabular}

de connaissances sur le cancer du col utérin, sur le fait qu'il est considéré comme une infection transmissible sexuellement et sur le dépistage. Les résultats de l'étude menée au Nigéria ont indiqué que la vaste majorité des femmes n'avaient jamais entendu parler du VPH (Bang et al., 2011). L'analyse préliminaire effectuée au Nigéria chez des femmes d'âge universitaire a révélé que les femmes qui avaient passé un test Pap avaient plus de connaissances sur la santé du col utérin que celles qui n'en avaient pas passé (Gimba, Emmanuel, Afoi, Manai et Bukuta, 2014). Dans une étude effectuée au Zimbabwe par Mupepi, Sampselle et Johnson (2011), 81 \% des 514 participantes ne connaissaient pas ce test de dépistage.

L'Organisation mondiale de la Santé (WHO/OMS, 2019) a confirmé que beaucoup de femmes et d'hommes n'avaient jamais entendu parler du cancer du col utérin et n'avaient aucune idée des signes et des symptômes. De plus, comme les femmes à risque manquent de connaissances à ce sujet, elles ne savent peut-être pas que le test est nécessaire

\section{Tableau 2b : Attitude des répondantes sur le dépistage du cancer} du col utérin

\begin{tabular}{|l|c|c|c|c|}
\hline Attitude & Oui & Non & $\begin{array}{c}\text { Je ne sais } \\
\text { pas }\end{array}$ & Total \\
\hline $\begin{array}{l}\text { Êtes-vous } \\
\text { intéressée à } \\
\text { participer au } \\
\text { dépistage? }\end{array}$ & $237(98,8)$ & $3(1,3)$ & $0(0,0)$ & $\begin{array}{c}240 \\
(100)\end{array}$ \\
\hline $\begin{array}{l}\text { Encouragerez- } \\
\text { vous d'autres } \\
\text { femmes ày } \\
\text { participer? }\end{array}$ & $\begin{array}{c}240 \\
(100,0)\end{array}$ & $0(0,0)$ & $0(0,0)$ & $\begin{array}{c}240 \\
(100)\end{array}$ \\
\hline $\begin{array}{l}\text { Est-ce que le } \\
\text { cancer vous } \\
\text { effraie? }\end{array}$ & $174(72,5)$ & $66(27,5)$ & $0(0,0)$ & $\begin{array}{c}240 \\
(100)\end{array}$ \\
\hline $\begin{array}{l}\text { Croyez-vous } \\
\text { etre susceptible } \\
\text { de développer } \\
\text { le cancer du col } \\
\text { utérin? }\end{array}$ & $67(27,9)$ & $\begin{array}{c}158 \\
(65,8)\end{array}$ & $15(6,3)$ & $\begin{array}{c}240 \\
(100)\end{array}$ \\
\hline $\begin{array}{l}\text { Le dépistage } \\
\text { du cancer du } \\
\text { col utérin est-il } \\
\text { important pour } \\
\text { vous? }\end{array}$ & $199(82,9)$ & $20(8,3)$ & $21(8,8)$ & $\begin{array}{c}240 \\
(100)\end{array}$ \\
\hline $\begin{array}{l}\text { Se rendre à } \\
\text { un test de Pap } \\
\text { coûte trop cher. }\end{array}$ & $137(57,1)$ & 100 & $3(1,3)$ & $(100)$ \\
\hline $\begin{array}{l}\text { J'ai peur d'avoir } \\
\text { un résultat } \\
\text { anormal. }\end{array}$ & $117(48,8)$ & $\begin{array}{c}123 \\
(51,3)\end{array}$ & $0(0,0)$ & 240 \\
\hline
\end{tabular}




\begin{tabular}{|c|c|c|}
\hline \multicolumn{3}{|c|}{$\begin{array}{l}\text { Tableau } 3 \text { : Croyances en matière de santé sur le dépistage du cancer du } \\
\text { col utérin }\end{array}$} \\
\hline Croyances en matière de santé & Fréquence & Pourcentage \\
\hline \multicolumn{3}{|l|}{$\begin{array}{l}\text { Pensez-vous avoir une lésion } \\
\text { précancéreuse? }\end{array}$} \\
\hline Oui & 82 & 34,2 \\
\hline Non & 99 & 41,3 \\
\hline Je ne sais pas & 59 & 24,6 \\
\hline Total & 240 & 100 \\
\hline \multicolumn{3}{|l|}{$\begin{array}{l}\text { Selon vous, le cancer du col utérin est-il une } \\
\text { préoccupation de santé publique? }\end{array}$} \\
\hline Oui & 228 & 95,0 \\
\hline Non & 12 & 5,0 \\
\hline Total & 240 & 100 \\
\hline \multicolumn{3}{|l|}{$\begin{array}{l}\text { Croyez-vous qu'il y a des chances qu'on } \\
\text { découvre un traitement contre le cancer du } \\
\text { col utérin? }\end{array}$} \\
\hline De bonnes chances & 119 & 49,6 \\
\hline Aucune chance & 78 & 32,5 \\
\hline Je ne sais pas & 43 & 17,9 \\
\hline Total & 240 & 100 \\
\hline \multicolumn{3}{|l|}{ Croyez-vous qu'on peut guérir ce cancer? } \\
\hline Oui & 119 & 49,6 \\
\hline Non & 112 & 46,7 \\
\hline Je ne sais pas & 9 & 3,8 \\
\hline Total & 240 & 100 \\
\hline \multicolumn{3}{|l|}{$\begin{array}{l}\text { Croyez-vous que le cancer du col est une } \\
\text { malédiction divine? }\end{array}$} \\
\hline Oui & 1 & 0,4 \\
\hline Non & 232 & 96,7 \\
\hline Je ne sais pas & 7 & 2,9 \\
\hline Total & 240 & 100 \\
\hline \multicolumn{3}{|l|}{$\begin{array}{l}\text { Serez-vous satisfaite après avoir subi un test } \\
\text { Pap pour dépister le cancer du col utérin? }\end{array}$} \\
\hline Oui & 217 & 90,4 \\
\hline Non & 3 & 1,3 \\
\hline Je ne sais pas & 20 & 8,3 \\
\hline Total & 240 & 100 \\
\hline \multicolumn{3}{|l|}{$\begin{array}{l}\text { Obtenir un résultat normal vous donne-t-il } \\
\text { un sentiment de contrôle? }\end{array}$} \\
\hline Oui & 189 & 78,8 \\
\hline Non & 21 & 8,8 \\
\hline Je ne sais pas & 30 & 12,5 \\
\hline Total & 240 & 100 \\
\hline \multicolumn{3}{|l|}{$\begin{array}{l}\text { À votre avis, un test Pap devrait-il être } \\
\text { effectué régulièrement? }\end{array}$} \\
\hline Oui & 191 & 79,6 \\
\hline Non & 41 & 17,1 \\
\hline Je ne sais pas & 8 & 3,3 \\
\hline Total & 240 & 100 \\
\hline
\end{tabular}

même en l'absence de symptôme (OMS, 2019). Ceci laisse croire que la plupart des femmes qui ont participé au dépistage sont celles qui ont des connaissances sur la maladie. En outre, l'appareil génital féminin est souvent considéré comme intime, et les femmes se sentent parfois intimidées de discuter des symptômes qui y sont reliés (OMS, 2019). Déstigmatiser les discussions sur l'appareil génital féminin à l'aide de la sensibilisation constituerait une stratégie importante pour encourager les femmes à se faire dépister et à chercher des soins si elles ont des symptômes associés au cancer du col utérin. Les répondantes de la présente étude n'avaient pas de connaissances approfondies sur le cancer du col utérin et le dépistage, provoquant possiblement un faible achalandage du service.

La plupart des femmes de l'étude ne connaissaient pas le cancer du col utérin et n'avaient pas de connaissances précises sur son dépistage. Ceci vient corroborer d'autres études internationales qui ont montré que les femmes n'ont pas suffisamment de connaissances sur les facteurs de risque du cancer du col utérin et le test Pap (Saha, Chaudhury, Bhowmik et Chatterjee, 2010). L'OMS (2019) a rapporté que, à plusieurs endroits, la mise en place d'une sensibilisation avait été ignorée par les décideurs, les prestataires de soins de santé et la population tout entière, entraînant des connaissances inadéquates sur la maladie et les mesures préventives.

Plusieurs études, dont une étude réalisée dans un collège de femmes par Baer, Allen et Braun (2000) a confirmé que la majorité des étudiantes ignoraient ce qu'étaient l'infection du col, les moyens de transmission et la prévalence dans la société. Faisant écho aux répondantes de la présente étude, les connaissances sur le VPH étaient aussi lacunaires : moins de la moitié ont su identifier le VPH comme cause principale de ce cancer. Ces répondantes doivent être renseignées sur les divers aspects de la maladie et sur les nouvelles méthodes de dépistage et le test. Beaucoup d'études montrent que les connaissances des femmes avaient augmenté après une séance d'information sur la prévention du cancer du col utérin et qu'elles étaient plus enclines ensuite à passer un test Pap ou à vouloir en passer un (Yücel, Ceber et Özentürk, 2009).

Dans cette étude, la plupart des répondantes ont rapporté qu'il y a des risques d'avoir un cancer du col utérin entre 18 et 25 ans et que le dépistage est nécessaire. Ceci est cohérent avec une étude menée par le United States Preventive Task Force (2018) qui a indiqué que le dépistage de ce cancer chez les femmes de ce groupe d'âge réduisait substantiellement l'incidence du cancer du col utérin et la mortalité. Ces résultats sont aussi cohérents avec une étude menée à Accra, capitale du Ghana, en 2009, parmi des étudiantes du collégial. La majorité des participantes à cette étude étaient intéressées au dépistage parce qu'elles étaient actives sexuellement et qu'ainsi elles s'exposaient au virus. C'est pourquoi il est nécessaire de faire davantage de sensibilisation sur le test Pap afin de prévenir le cancer du col utérin et de poser un diagnostic précoce. De plus, une majorité de répondantes ne savaient pas le délai normal de suivi après un résultat normal. En fait, 
l'American Cancer Society recommande un suivi trois ans après un résultat normal.

Du côté des comportements, moins de $10 \%$ des répondantes de la présente étude avaient subi un test Pap. Lâge et le niveau de scolarité semblaient avoir une incidence sur cette réponse. Les études indiquent un lien positif entre le niveau de scolarité et l'attitude en matière d'initiatives sur la santé; de plus grandes connaissances sont associée à une augmentation du dépistage. Étant donné le peu de connaissances sur le test Pap, le faible taux de dépistage parmi les répondantes de la présente étude n'est pas étonnant. Le Health Belief Model indique que les femmes qui sont les plus susceptibles de passer un test Pap sont celles qui pensent être vulnérables au cancer du col utérin, qui croient à l'efficacité du test, qui savent que la procédure est sans douleur, et qui sont aiguillées par un professionnel de la santé (Rosenstock, Strecher et Becker, 1994). La majorité des répondantes ont rapporté qu'elles avaient peur du cancer et des résultats du test. Des études ont montré que trop de connaissances sur le cancer du col utérin suscitaient parfois une anxiété accrue, et diminuait donc l'adhésion au dépistage (Blomberg, Forss, Ternestedt et Tishelman, 2009). De la même manière, 57,1 \% des répondantes étaient d'avis que le dépistage est dispendieux, ce qui en limitait le recours. Au Ghana, une étude effectuée par Ampofo (2020) a confirmé que l'utilisation du dépistage était significativement affectée par les coûts élevés.

Les répondantes de cette étude qui ne croyaient pas pouvoir avoir des lésions précancéreuses étaient moins portées vers le dépistage et moins intéressées à comprendre la maladie. Les résultats pourraient venir du fait que ces femmes croyaient que si elles n'avaient pas de signes et de symptômes de la maladie, le dépistage n'était pas très important. Par ailleurs, dans cette étude, une majorité de répondantes percevaient le cancer du col utérin comme un enjeu de santé publique. Cette perception est d'une importance fondamentale, car elle influencera positivement l'attitude de ces femmes à agir pour freiner

\section{RÉFÉRENCES}

Adanu, R. M. K., Seffah, J. D., Duda, R., Darko, R., Hill, A., \& Anarfi, J. (2010). Clinic visits and cervical cancer screening in Accra. Ghana medical journal, 44(2).

Adenola, F., \& Saibu, O. M. (2017). Does population change matter for long run economic growth in Nigeria. International Journal of Development and Sustainability, 12(6), 1955-1965.

Ampofo, A. G., Adumatta, A. D., Owusu, E., \& Awuviry-Newton, K. (2020). A cross-sectional study of barriers to cervical cancer screening uptake in Ghana: An application of the health belief model. PloS one, 15(4), e0231459

Baer, H., Allen, S., \& Braun, L. (2000). Knowledge of human papillomavirus infection among young adult men and women: implications for health education and research. Journal of community health, 25(1), 67-78.

Bang, K. S., Sung, S., Koo, B., Kim, M., Kim, Y., Kim, J., \& Ryu, S. (2011). Female university students' HPV-related knowledge and influencing factors on HPV vaccination. Journal of Korean Oncology Nursing, 11(3), 186-192. cette maladie. L'analyse des données d'une étude menée aux Pays-Bas (Tacken et al., 2007) a montré que les croyances des femmes sur le dépistage du cancer du col utérin constituent le meilleur prédicteur du recours au dépistage, même lorsque les aspects démographiques et organisationnels sont pris en compte.

Basées sur les conclusions de l'étude, les mesures suivantes sont proposées :

1. Les femmes du groupe d'âge pertinent, et particulièrement celles vivant en milieu rural, doivent être mieux renseignées sur les divers aspects du dépistage du cancer du col utérin et le test Pap. Les infirmières de santé publique des diverses communautés doivent recevoir de la formation sur le cancer du col utérin pour aider à la sensibilisation dans les écoles durant les rencontres communautaires.

2. Des politiques pour améliorer l'accès au test de dépistage doivent être mises en place pour s'assurer d'une plus grande protection chez les femmes.

3. Le dépistage du cancer du col utérin et le test Pap doivent être intégrés au programme des établissements tertiaires pour accroître la sensibilisation et les connaissances des femmes à ce sujet.

4. Les décideurs doivent tenir compte de l'intégration des activités de dépistage du cancer du col utérin avec les services de santé primaire et accorder à la maladie les mêmes priorités qu'aux autres maladies comme la malaria, la tuberculose, le VIH et l'hypertension.

5. D'autres études sur le sujet devront être menées pour élargie la portée de l'étude $\mathrm{s}$ à d'autres districts.

\section{CONCLUSION}

Cette étude a démontré que les répondantes avaient peu de connaissances sur le dépistage du cancer du col utérin et cultivaient de fausses croyances en matière de santé sur cette maladie et son dépistage, facteurs qui ont influencé leurs attitudes envers l'adhésion au dépistage.

Blomberg, K., Forss, A., Ternestedt, B. M., \& Tishelman, C. (2009). From 'silent'to 'heard': Professional mediation, manipulation and women's experiences of their body after an abnormal Pap smear. Social science Q medicine, 68(3), 479-486.

Bruni L, Albero G, Serrano B, Mena M, Gómez D, Muñoz J, Bosch F X, de Sanjosé, S. (2019). Human Papillomavirus and related diseases in Ghana.

Ebu, N. I., Mupepi, S. C., Siakwa, M. P., \& Sampselle, C. M. (2015). Knowledge, practice, and barriers toward cervical cancer screening in Elmina, Southern Ghana. International Journal of Women's Health, 7, 31-39. https://doi.org/10.2147/IJWH.S71797

Ghana Districts. (2006). Ajumako-Enyan-Essiam district Assembly. Min of Local Government \& Rural Dev. and Maks Publications Q Media Services. http://aeeda.ghanadistricts.gov.gh

Gimba, S. M., Emmanuel, A., Afoi, B. B., Mangai, M. J., \& Bukuta, G. (2014). Awareness and practice of cervical cancer screening among University of Jos female undergraduates. Continental J. Nursing Science 6(1), $1-11$. 
Glenn, D. I. (1992). Determining sample size. A series of the Program Evaluation and Organizational Development. University of Florida. Publication date: November.

GSS, G., \& Macro, I. C. F. (2015). Ghana demographic and health survey 2014. Ghana Statistical Service, Ghana Health Service, and ICF International.

Mattocks, K. M., Kauth, M. R., Sandfort, T., Matza, A. R., Sullivan, J. C., \& Shipherd, J. C. (2014). Understanding healthcare needs of sexual and gender minority veterans: How targeted research and policy can improve health. LGBT health, 1(1), 50-57.

Mupepi, S. C., Sampselle, C. M., \& Johnson, T. R. (2011). Knowledge, attitudes, and demographic factors influencing cervical cancer screening behavior of Zimbabwean women. Journal of Women's Health, 20(6), 943-952.

Mukama, T., Ndejjo, R., Musabyimana, A., Halage, A. A., \& Musoke, D. (2017). Women's knowledge and attitudes towards cervical cancer prevention: A cross sectional study in Eastern Uganda. BMC women's health, 17(1), 1-8.

Rosenstock, I. M., Strecher, V. J., \& Becker, M. H. (1994). The health belief model and HIV risk behavior change. In Preventing AIDS (pp. 5-24). Springer.

Reis, N., Bebiş, H., Köse, S., Sis, A., Engin, R., \& Yavan, T. (2012). Knowledge, behavior and beliefs related to cervical cancer and screening among Turkish women.
Saha, A., Chaudhury, A. N., Bhowmik, P., \& Chatterjee, R. (2010). Awareness of cervical cancer among female students of premier colleges in Kolkata, India. Asian Pac J Cancer Prev, 11(4), 1085-90.

Tacken, M. A. J. B., Braspenning J. C. C., Hermans, R. P. M. G., et al. (2007). Uptake of cervical cancer screening in The Netherlands is mainly influenced by women's beliefs about the screening and by the inviting organization. Eur J Public Healh, 17(2), 178-85.

Tung, W. C., Lu, M., \& Cook, D. (2010). Papanicolaou screening in Taiwan: Perceived barriers and self-efficacy. Health care for women international, 31(5), 421-434.

US Preventive Services Task Force. (2003). Screening for cervical cancer: Recommendations and rationale. AJN The American Journal of Nursing, 103(11), 101-109.

Yücel, U., Ceber, E., \& Özentürk, G. (2009). Efficacy of a training course given by midwives concerning cervical cancer risk factors and prevention. Asian Pac J Cancer Prev, 10(3), 437-442.

World Health Organization. (2019). WHO framework for strengthening and scaling-up of services for the management of invasive cervical cancer. World Health Organization.

World Health Organization. (2013). Transforming and scaling up health professionals' education and training: World Health Organization guidelines 2013. World Health Organization. 